\title{
UEMS Section of Radiology Meeting with Delegates in Rom
}

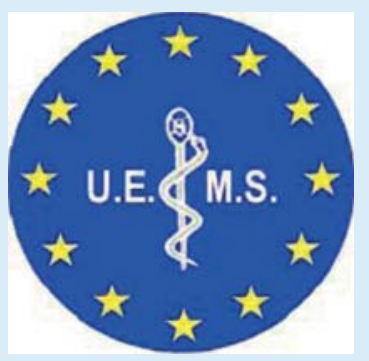

Die in der Überschrift genannte Abkürzung UEMS ist wohl nicht jedem Leser auf Anhieb präsent. Denn entgegen der beim Meeting gepflegten Konferenzsprache Englisch steht UEMS für das französische „Union Européenne des Médecins Spécialistes“, also die Europäische Vereinigung der Facharztverbände. Diese besitzt Sektiones, unter anderem für Radiologie, entsprechend einem europäischen Pendant zum Berufsverband der Radiologen. Über die letzte Delegiertenversammlung der Section of Radiology der UEMS, stattgefunden in Rom am 29. Oktober 2016 soll hier berichtet werden.

Die UEMS wurde 1958 als erster medizinischer Spitzenverband auf europäischer Ebene gegründet. Oberstes Entscheidungsgremium ist das sogenannte Council, das sich aus Vertretern der Mitgliedsländer zusammensetzt. Über die Vertreter entscheidet der entsendende nationale Facharztverband. Für Deutschland hat diese Aufgabe seit dem 1.1.2017 der Spitzenverband der Fachärzte (SpiFa) von der Gemeinschaft Fachärztlicher Berufsverbände (GFB) übernommen. Alle innerhalb der Sektiones erarbeiteten fachlichen Details müssen vom Council genehmigt bzw. bestätigt werden. Ähnlich nationaler Entscheidungsgremien wie z. B. dem Deutschen Ärztetag ist damit eine Mehrheitsfindung für radiologische Belange erforderlich.
Oberstes Ziel der UEMS ist die europaweite Harmonisierung der Facharztausbildungen, einheitliche Anerkennungskriterien für zertifizierte Fortbildungen und in Zukunft ein möglichst europaweites Qualitätsmanagement in der fachärztlichen Medizin. Hierzu sind eine Vielzahl nationaler Initiativen ebenso zu berücksichtigen, wie die europäischen Fachverbände der unterschiedlichen Arztgruppen. Für die Radiologie besteht eine enge Zusammenarbeit zwischen der European Society of Radiology (ESR) als wissenschaftliche Fachgesellschaft und der Section of Radiology der UEMS. Äußeres Zeichen hierfür ist ein räumlich und personell gemeinsames Sekretariat in Brüssel.

Die Section of Radiology der UEMS wurde anlässlich des ECR 2016 nach personellen Neubesetzungen deutlich gestärkt und die Delegiertenversammlung in Rom stellte eine Art kick-off-Meeting mit einer Vielzahl neu bestimmter Delegierter dar. Zahlenmäßig überdurchschnittlich vertreten waren die Länder Süd- und Süd-Ost-Europas sowie aus Skandinavien. Als Besonderheit ist zu erwähnen, dass die Section of Radiology über eine jeweils separate Division of Interventional Radiology und eine Division of Neuroradiology verfügt. Dennoch konnte in der Vergangenheit nicht verhindert werden, dass ein Curriculum für Angiologie auch im Council verabschiedet wurde, dass interventionelle Gefäßmaßnahmen als Kernbestandteil des Faches ohne Beteiligung der Radiologie vorsieht. Ähnlich nationalen Leitliniengremien gibt es auch auf europäischer Ebene Arbeitsgruppen, die Curricula unter Einschluss radiologischer Themenkomplexe ohne Beteiligung unserer Fachvertreter verabschieden. Es war einhellige Meinung der Delegierten, dass dies wenn immer verhindert werden sollte. Dazu ist jedoch eine weitreichende Mitarbeit in den Multidisciplinary Joint Committees (MJCS) und den Thematic Fe- derations (TF) erforderlich. Daher erging auch der Aufruf des Vorstandes der Sektion sich künftig intensiver an den entsprechenden Arbeitsgruppen zu beteiligen. Für die derzeit tätigen Arbeitsgruppen pädiatrische Urologie, Gefäßchirurgie, Phlebologie, Sportmedizin und Schmerztherapie sollen spätestens beim nächsten Treffen anlässlich des ECR 2017 in Wien entsprechende Vertreter der Sektion benannt werden.

Durch die seit 1.1.2017 übernommene EUPräsidentschaft Maltas sind neue europäische Initiativen auf dem Gesundheitssektor zu erwarten. So ist bereits angekündigt, dass die maltesische EU-Präsidentschaft Impulse bei der Harmonisierung einer hochqualitativen Gesundheitsversorgung, Facharztausbildung und einer grenzüberschreitenden Weiterbildung setzen möchte. Dies wird auch Gegenstand der nächsten Delegiertenversammlung beim ECR 2017 sein. Zusätzlich ist eine gemeinsame Veranstaltung der ESR und der Section of Radiology der UEMS beim ECR geplant.

Ein abschließendes gemeinsames Abendesssen aller Delegierten mit dem Vorstand der Sektion über den Hügeln von Rom bot die Möglichkeit des intensiven Meinungsaustausches und diente auch dem persönlichen Kennenlernen.

Fazit: Europäische Berufspolitik ist in seiner Tiefe sicherlich nochmals deutlich komplexer als nationale und Ergebnisse ein Produkt langwieriger Prozesse. Auch scheint die Vision einer qualitativ und fachlich einheitlichen europaweiten Patientenversorgung aus heutiger Sicht weit entfernt und möglicherweise aus nationaler Sicht Deutschlands weniger relevant. Die Bedeutung europäischer Vorgaben, die dann auch in nationales Recht umzusetzen sind, ist allerdings nicht zu unterschätzen. Vergleichbare Beispiele liefern die Diskussionen um 
das Atomrecht und die damit verbundene Röntgenverordnung oder die Diskussionen um die Sicherheit der MRT. Auch wenn die Radiologie im Vergleich zu den anderen Fächern, wie immer, ein Juniorpartner bleibt, wäre es fatal nicht vertreten zu sein. Die Neustrukturierungen innerhalb der Section of Radiology der UEMS, der UEMS selbst, aber auch bei den deutschen Ansprechpart- nern, die die Vertreter in das Council der UEMS entsenden, sollten genutzt werden, um gemeinsam mit der ESR als Vertreterin der wissenschaftlichen radiologischen Fachgesellschaften die Zukunft unseres Faches zu sichern und zu entwickeln.

Prof. Helmberger und Prof. Maintz vertreten in 2017 die Radiologie bei der UEMS
Über die Autoren

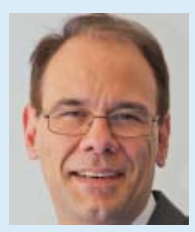

Prof. Hermann Helmberger, München 